\title{
A Block Cipher Involving a Key Bunch Matrix and Including another Key Matrix Supported With Modular Arithmetic Addition
}

\author{
V.U.K. Sastry, PhD. \\ Professor of CSE, Director (SCSI), \\ Dean (Admin), Dean (R\&D), \\ SreeNidhi Institute of Science \& Technology, \\ Hyderabad, India,
}

\begin{abstract}
In this paper, we have developed a block cipher, by using a key bunch matrix and an additional key matrix. In order to carry out the decryption process, the decryption key bunch matrix is obtained, basing upon the encryption key bunch matrix, on using the concept of multiplicative inverse. In the cryptanalysis, we have found that, this cipher is a very strong one as it includes the additional key matrix and supported with modular arithmetic addition. From the viewpoint of efficiency and strength, this cipher is quite comparable with any other cipher available in the literature of cryptography.
\end{abstract}

\section{Keywords}

encryption key bunch matrix, decryption key bunch matrix, additional key matrix, cryptanalysis, avalanche effect.

\section{INTRODUCTION}

Transmission of information through internet has been a fascinating area of research, as every secret information is to be maintained in a secured manner. In a recent development [1-2], we have developed a novel block cipher by including a key bunch matrix for encryption, and extended the analysis by introducing another key matrix supplemented with xor operation. In this analysis the decryption matrix is obtained from the given encryption matrix by using the concept of multiplicative inverse.

In the present paper, our objective is to develop a block cipher by using a key bunch matrix and introducing another key matrix by associating it with modular arithmetic addition. This cipher is expected to be very strong as we have a pair of keys in this analysis and the operations are supported by modular arithmetic addition.

The basic equation governing the encryption of a cipher is given by

$\mathrm{C}=\left(\left[\mathrm{e}_{\mathrm{ij}} \times \mathrm{p}_{\mathrm{ij}}\right] \bmod 256+\mathrm{F}\right) \bmod 256, \mathrm{i}=1$ to $\mathrm{n}, \mathrm{j}=1$ to $\mathrm{n}$.

The corresponding equation describing the decryption process is given by

$\mathrm{P}=\left[\mathrm{d}_{\mathrm{ij}} \times(\mathrm{C}-\mathrm{F})_{\mathrm{ij}}\right] \bmod 256, \mathrm{i}=1$ to $\mathrm{n}, \mathrm{j}=1$ to $\mathrm{n}$.

Here, $P=\left[p_{i j}\right]$ is the plaintext, $C=\left[c_{i j}\right]$, the ciphertext, $F=\left[f_{i j}\right]$ is an additional key matrix, whose elements are in [0-255]. The $\left[\mathrm{e}_{\mathrm{ij}}\right]$ are the elements of the encryption key bunch matrix $\mathrm{E}$, and $\left[\mathrm{d}_{\mathrm{ij}}\right]$ are the elements of the decryption key bunch matrix D. It is to be noted here that the $\left[\mathrm{e}_{\mathrm{ij}}\right]$ and the $\left[\mathrm{d}_{\mathrm{ij}}\right]$ are

\author{
K. Shirisha \\ Associate Professor, \\ Dept. of Computer Science \& Engineering, \\ SreeNidhi Institute of Science \& Technology, \\ Hyderabad, India,
}

odd integers lying in the interval [1,255] and they are governed by the relation

$$
\left(e_{i j} \times d_{i j}\right) \bmod 256=1 .
$$

For every given $e_{i j}$ we have a unique $d_{i j}$. Here our interest is to see, how the additional key $\mathrm{F}$ and modular arithmetic addition will add to the strength of the cipher.

In what follows, we present the plan of the paper. In section 2, we introduce the development of the cipher, and draw flowcharts and design algorithms for this cipher. We illustrate the cipher with a suitable example in section 3 , and then analyze the avalanche effect. We examine the cryptanalysis in section 4 . Finally in section 5 , we discuss the computations carried out in this investigation and draw conclusions.

\section{DEVELOPMENT OF THE CIPHER}

We consider a plaintext P. On using the EBCDIC code, this can be written in the form

$$
P=\left[p_{i j}\right], i=1 \text { to } n, j=1 \text { to } n .
$$

The encryption key bunch matrix $\mathrm{E}$ is given by

$$
\mathrm{E}=\left[\mathrm{e}_{\mathrm{ij}}\right], \mathrm{i}=1 \text { to } \mathrm{n}, \mathrm{j}=1 \text { to } \mathrm{n} \text {. }
$$

Let us have the additional key matrix $\mathrm{F}$ in the form

$$
F=\left[f_{i j}\right], i=1 \text { to } n, j=1 \text { to } n .
$$

We take the ciphertext $\mathrm{C}$ in the form

$$
\mathrm{C}=\left[\mathrm{c}_{\mathrm{ij}}\right], \mathrm{i}=1 \text { to } \mathrm{n}, \mathrm{j}=1 \text { to } \mathrm{n} .
$$

Let us take the decryption matrix D in the form

$$
D=\left[d_{i j}\right], i=1 \text { to } n, j=1 \text { to } n .
$$

The flowcharts for the encryption and decryption can be drawn in the form shown in figure 1 and figure 2, respectively.

The corresponding algorithms for the encryption and the decryption are given below.

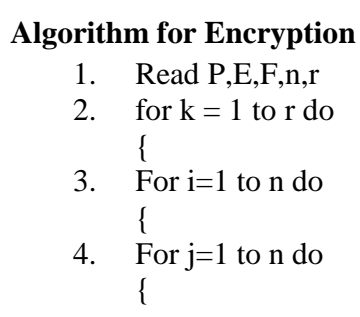




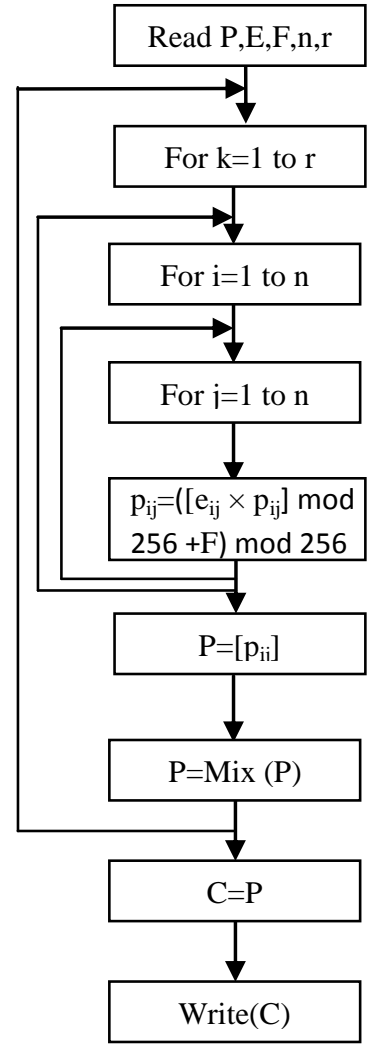

Figure 1. Flowchart for Encryption

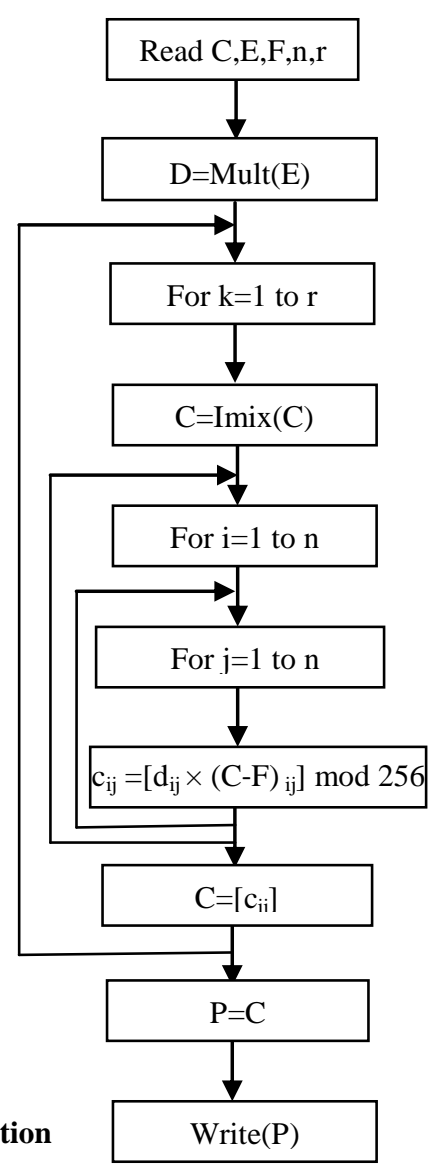

Figure 2. Flowchart for Decryption

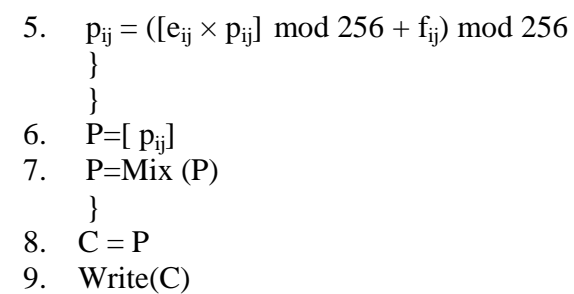

Algorithm for Decryption

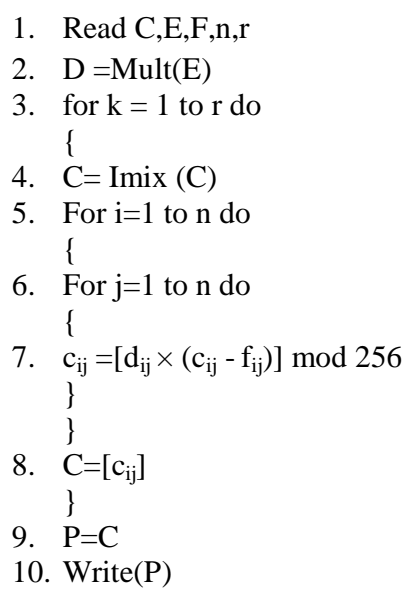

The number of rounds in the iteration process of this analysis are taken as $r=16$.

In the procedure for encryption, we have made use of the function $\operatorname{Mix}()$. The basic ideas underlying in this function can be outlined as follows.
Let $P=\left[p_{i j}\right], i=1$ to $n, j=1$ to $n$, be the plaintext in any round of the iteration process. Let us assume that $\mathrm{n}=2 \mathrm{~m}$. Then this matrix can be viewed as two sub-matrices, of equal size, wherein the first one is containing $\mathrm{n}$ rows and $\mathrm{m}$ columns, and the second one is also containing $\mathrm{n}$ rows and $\mathrm{m}$ columns (from $(m+1)$ th column to nth column). Now, we represent all the elements in both the matrices in their binary form. Thus each element is written in terms of eight binary bits. Now, taking the first eight bits in the first column, we write them in the form of a decimal number. We carry out the same process with the subsequent elements of the same column (if $n>8$ ). Then we perform the same operations with the binary bits of the first column of the second sub-matrix. Similarly, the process is repeated with the binary bits of the first and second sub-matrices till we exhaust all the columns. In other words, summarizing all this process, we can say that we are concatenating the binary bits of the first sub-matrix with the binary bits of the first column of the second sub-matrix and then the binary bits of the second column of the first submatrix with the second column of the second sub-matrix, and obtaining a string of binary bits. Then taking eight binary bits at each instance from the beginning, we write them in terms of decimal numbers, and arrange them in a square matrix of size $\mathrm{n}$ in a row-wise manner. This function $\operatorname{Mix}()$ is utilized to create confusion and diffusion. The function $\operatorname{Imix}()$, used in the decryption process, contains all the reverse operations of $\operatorname{Mix}()$.

The function Mult(), used in the decryption process, is intended to obtain the decryption key bunch matrix $\mathrm{D}$ for a given encryption key bunch matrix $\mathrm{E}$.

\section{ILLUSTRATION OF THE CIPHER AND THE AVALANCHE EFFECT}

Consider the plaintext given below.

Dear Brother! At the time of partition our grandfather felt that our family migrating to Karachi is the best one as we are expected to be very happy among our own community. Your father felt that he must continue his stay in Hyderabad as our ancestors earned a lot of property there. I know, to-day the size of your family is very large consisting of fifty or sixty members, you cannot come to us. We have to bear this life. Be informing about your welfare as frequently as possible.

We focus out attention on the first 16 characters of the above plaintext. Thus we have

Dear Brother! At

On using EBCDIC code, we get

$$
P=\left[\begin{array}{llll}
196 & 133 & 129 & 153 \\
64 & 194 & 153 & 150 \\
163 & 136 & 133 & 153 \\
79 & 64 & 193 & 163
\end{array}\right]
$$

Let us take the encryption key bunch matrix $\mathrm{E}$ in the form

$$
E=\left[\begin{array}{cccc}
39 & 29 & 205 & 177 \\
87 & 35 & 81 & 137 \\
233 & 119 & 69 & 247 \\
1 & 59 & 193 & 203
\end{array}\right]
$$

We have the additional key matrix $\mathrm{F}$ in the form given in (3.3). 


$F=\left[\begin{array}{llll}111 & 23 & 56 & 80 \\ 66 & 45 & 26 & 99 \\ 23 & 56 & 98 & 33 \\ 22 & 55 & 34 & 78\end{array}\right]$

Let us obtain the decryption key bunch matrix D by using the relation (1.3). Thus we have

$D=\left[\begin{array}{llll}151 & 53 & 5 & 81 \\ 103 & 139 & 177 & 185 \\ 89 & 247 & 141 & 199 \\ 1 & 243 & 65 & 227\end{array}\right]$

On using $\mathrm{P}, \mathrm{E}$ and $\mathrm{F}$, given by (3.1) to (3.3), and the encryption algorithm given in section 2 , we get

$$
C=\left[\begin{array}{cccc}
77 & 95 & 27 & 148 \\
246 & 164 & 248 & 153 \\
122 & 82 & 250 & 38 \\
95 & 198 & 69 & 147
\end{array}\right]
$$

On using the above $\mathrm{C}$, given by (3.5), the decryption key bunch matrix $\mathrm{D}$, given by (3.4), and the decryption algorithm given in section 2 , we get back the original plaintext $\mathrm{P}$, given by (3.1).

Now let us examine the avalanche effect. On changing 2nd row 3rd column element of the plaintext $\mathrm{P}$, given by (3.1), from 153 to 152 , we get a one bit binary change in the plaintext. On using, the modified plaintext, the key matrices, given by (3.2) and (3.3), and the encryption algorithm, given in section 2 , we get the ciphertext $\mathrm{C}$ in the form

$$
C=\left[\begin{array}{cccc}
178 & 114 & 17 & 165 \\
210 & 238 & 7 & 99 \\
132 & 207 & 17 & 216 \\
229 & 178 & 127 & 27
\end{array}\right]
$$

On comparing (3.5) and (3.6), after representing them in their binary form, we notice that these two ciphertexts differ by 76 bits out of 128 bits.

Now, let us have a one bit change in the encryption key bunch matrix E. To this end, we change the 1 st row 3rd column element of (3.2), from 205 to 201. On using this modified E, the plaintext $\mathrm{P}$, given by (3.1), the additional key matrix $\mathrm{F}$, given by (3.3), and using the encryption algorithm, we get the ciphertext $\mathrm{C}$ in the form

$$
C=\left[\begin{array}{cccc}
26 & 232 & 4 & 12 \\
72 & 45 & 68 & 161 \\
16 & 8 & 20 & 202 \\
3 & 94 & 183 & 187
\end{array}\right]
$$

On comparing, (3.7) and (3.5), in their binary form, we find that they differ by 69 bits out of 128 bits.

From the above analysis, we conclude that the avalanche effect is conspicuous.

\section{CRYPTANALYSIS}

In the development of every cipher, cryptanalysis play a fundamental role as it decides whether a cipher is having strength or not. The different types of attacks that are carried out in cryptanalysis are

1. Ciphertext only attack (Brute force attack),

2. Known plaintext attack,

3. Chosen plaintext attack, and
4. Chosen ciphertext attack.

Generally every cipher is designed [3] so that it sustains the first two attacks.

Let us firstly analyze the ciphertext only attack. In this analysis, the key bunch matrix $\mathrm{E}$ is containing only odd integers lying in [1-255], and the additional key matrix $\mathrm{F}$ is containing integers lying in [0-255]. In the light of this fact, the size of the key space is

$$
2^{15 n^{2}}=\left(2^{10}\right)^{1.5 n^{2}} \approx\left(10^{3}\right)^{1.5 n^{2}}=10^{4.5 n^{2}} .
$$

If we assume that the time required for the computation of this cipher with one $\mathrm{E}$ and one $\mathrm{F}$ in the key space is $10^{-7}$ seconds, then the time required for the execution of the cipher with all the keys in the key space is approximately equal to

$$
\frac{10^{4.5 n^{2}} \times 10^{-7}}{365 \times 24 \times 60 \times 60}=3.12 \times 10^{4.5 n^{2}-15} \text { years } .
$$

In this analysis, as we have taken $n=4$, the time required can be written in the form $3.12 \times 10^{57}$ years.

As this time is very large, it is not at all possible to break this cipher by the ciphertext only attack.

Let us now examine the known plaintext attack. In order to carry out this attack, the attacker knows as many plaintexts as he wants, and the corresponding ciphertexts. Having this entire bunch, his job is to break this cipher if possible.

If we restrict our attention to the first round of iteration process, (that is $r=1$ ) the equations governing the encryption process (see the algorithm in section 2) are given by

$$
\begin{aligned}
& \mathrm{P}=\left(\left[\mathrm{e}_{\mathrm{ij}} \times \mathrm{p}_{\mathrm{ij}}\right] \bmod 256+\mathrm{F}\right) \bmod 256, \mathrm{i}=1 \text { to } \mathrm{n}, \mathrm{j}=1 \text { to } \mathrm{n},(4.1) \\
& \mathrm{P}=\mathrm{Mix}(\mathrm{P}), \\
& \text { and } \\
& \mathrm{C}=\mathrm{P}
\end{aligned}
$$

In this system of equations, the $\mathrm{p}_{\mathrm{ij}}$ occurring in (4.1), and the ciphertext in (4.3) are known to us. As $\mathrm{C}$ is known, the $\mathrm{P}$ occurring left hand side of (4.2) is available. On using $\operatorname{Imix}()$ operation, the $\mathrm{P}$ occurring on the right hand side of (4.2) and hence the P occurring on the left hand side of (4.1) can be determined. As the equation (4.1) contains the additional key matrix $\mathrm{F}$, the $\mathrm{e}_{\mathrm{ij}}$ cannot be determined by any means. Hence this cipher cannot be broken by this attack. This is the situation when $r=1$. Here in this analysis, as we have taken $\mathrm{r}=16$, we can say very firmly that this cipher cannot be broken by the known plaintext attack

Intuitively, we notice that, we cannot choose either a plaintext or a ciphertext in a convenient manner to attack this cipher.

\section{COMPUTATIONS AND CONCLUSIONS}

In this analysis, we have developed a block cipher by using two key matrices. The first matrix, E, contains a bunch of keys, where in each key is an odd integer which lies in [1255], while the second one, $\mathrm{F}$, includes integers which lie in [0-255]. The cryptanalysis carried out in this investigation has clearly indicated that this cipher is a strong one and it cannot be broken by any cryptanalytic attack.

The programs for encryption and decryption are written in Java.

The plaintext given in (3.1) is divided into 31 blocks, where in each block is having 16 characters. The last block which contains three characters is made a complete block by 
appending thirteen 0 s as characters. On using, E and F, and the encryption algorithm, given in section 2 , we have obtained the ciphertext for all blocks. Here, we have presented the ciphertext corresponding to all these blocks (excluding the ciphertext of the first block) in (5.1).

The strength of the cipher has increased significantly on account of the introduction of additional key matrix, F, and the modular arithmetic addition operation. This cipher which we have developed in this analysis is quite comparable with any other cipher available in the literature of cryptography.

\section{REFERENCES}

[1] V.U.K. Sastry, K. Shirisha, "A Novel Block Cipher Involving a Key Bunch Matrix" sent for publication.

[2] V.U.K. Sastry, K. Shirisha, "A Block Cipher Involving a Key Bunch Matrix and Including another Key Matrix Supplemented with Xor Operation" sent for publication.

[3] William Stallings, "Cryptography and Network Security: Principle and Practices", Third Edition 2003, Chapter 2, pp. 29.

\begin{tabular}{|c|c|c|c|c|c|c|c|c|c|c|c|c|c|c|c|}
\hline 80 & 132 & 150 & 8 & 10 & 19 & 83 & 66 & 214 & 133 & 104 & 238 & 184 & 238 & 205 & 201 \\
\hline 254 & 32 & 238 & 228 & 255 & 62 & 242 & 131 & 149 & 130 & 202 & 77 & 69 & 133 & 71 & 162 \\
\hline 10 & 17 & 71 & 12 & 26 & 232 & 49 & 9 & 63 & 51 & 134 & 121 & 52 & 146 & 228 & 117 \\
\hline 63 & 229 & 53 & 240 & 75 & 218 & 14 & 92 & 41 & 218 & 41 & 197 & 82 & 71 & 79 & 47 \\
\hline 119 & 32 & 196 & 119 & 52 & 204 & 98 & 136 & 117 & 242 & 72 & 177 & 236 & 203 & 58 & 65 \\
\hline 169 & 204 & 14 & 92 & 248 & 49 & 94 & 63 & 112 & 127 & 106 & 145 & 249 & 98 & 178 & 49 \\
\hline 26 & 119 & 187 & 213 & 137 & 196 & 154 & 122 & 217 & 35 & 131 & 210 & 193 & 181 & 29 & 111 \\
\hline 39 & 208 & 212 & 252 & 121 & 188 & 211 & 123 & 187 & 45 & 48 & 95 & 55 & 0 & 118 & 252 \\
\hline 182 & 152 & 169 & 54 & 90 & 52 & 163 & 224 & 126 & 118 & 25 & 67 & 215 & 68 & 187 & 68 \\
\hline 31 & 236 & 218 & 48 & 235 & 8 & 100 & 19 & 39 & 176 & 5 & 19 & 225 & 17 & 244 & 242 \\
\hline 91 & 36 & 86 & 12 & 160 & 25 & 171 & 75 & 199 & 77 & 175 & 5 & 102 & 4 & 147 & 195 \\
\hline 25 & 12 & 205 & 102 & 89 & 124 & 206 & 110 & 202 & 16 & 123 & 43 & 75 & 76 & 99 & 37 \\
\hline 162 & 198 & 119 & 56 & 41 & 216 & 120 & 250 & 104 & 26 & 119 & 236 & 50 & 154 & 18 & 111 \\
\hline 253 & 177 & 106 & 150 & 139 & 181 & 226 & 226 & 178 & 35 & 80 & 119 & 206 & 235 & 77 & 216 \\
\hline 50 & 216 & 184 & 99 & 53 & 226 & 254 & 216 & 85 & 91 & 83 & 84 & 122 & 117 & 224 & 127 \\
\hline 156 & 121 & 130 & 68 & 193 & 60 & 169 & 90 & 40 & 209 & 223 & 123 & 180 & 156 & 235 & 195 \\
\hline 56 & 166 & 22 & 236 & 175 & 187 & 176 & 149 & 189 & 29 & 126 & 31 & 167 & 10 & 226 & 255 \\
\hline 231 & 102 & 254 & 48 & 82 & 162 & 214 & 148 & 78 & 196 & 14 & 239 & 246 & 37 & 39 & 86 \\
\hline 99 & 193 & 223 & 165 & 71 & 176 & 87 & 232 & 136 & 198 & 0 & 30 & 60 & 209 & 27 & 195 \\
\hline 80 & 129 & 144 & 125 & 236 & 143 & 129 & 78 & 244 & 251 & 243 & 152 & 204 & 38 & 170 & 235 \\
\hline 104 & 121 & 126 & 62 & 143 & 81 & 0 & 222 & 120 & 191 & 133 & 61 & 149 & 70 & 189 & 224 \\
\hline 181 & 122 & 33 & 58 & 56 & 103 & 211 & 157 & 22 & 252 & 126 & 71 & 24 & 238 & 187 & 123 \\
\hline 60 & 154 & 198 & 228 & 95 & 137 & 253 & 77 & 62 & 2 & 6 & 143 & 191 & 107 & 86 & 132 \\
\hline 249 & 168 & 197 & 100 & 213 & 109 & 21 & 27 & 163 & 118 & 150 & 49 & 159 & 181 & 42 & 71 \\
\hline 155 & 130 & 67 & 50 & 222 & 171 & 67 & 115 & 94 & 9 & 12 & 36 & 227 & 247 & 196 & 240 \\
\hline 78 & 189 & 201 & 242 & 54 & 222 & 201 & 128 & 8 & 89 & 112 & 168 & 104 & 136 & 103 & 58 \\
\hline 104 & 172 & 255 & 35 & 15 & 158 & 233 & 131 & 77 & 177 & 109 & 136 & 10 & 67 & 89 & 179 \\
\hline 223 & 174 & 129 & 108 & 59 & 2 & 42 & 51 & 240 & 178 & 86 & 119 & 217 & 244 & 55 & 94 \\
\hline 30 & 18 & 49 & 239 & 235 & 23 & 214 & 23 & 98 & 94 & 59 & 87 & 216 & 23 & 132 & 98 \\
\hline 169 & 5 & 8 & 10 & 170 & 196 & 243 & 229 & 127 & 155 & 185 & 162 & 196 & 142 & 247 & 72 \\
\hline
\end{tabular}

\section{AUTHORS PROFILE}

Dr. V. U. K. Sastry is presently working as Professor in the Dept. of Computer Science and Engineering (CSE), Director (SCSI), Dean (R \& D), SreeNidhi Institute of Science and Technology (SNIST), Hyderabad, India. He was Formerly Professor in IIT, Kharagpur, India and worked in IIT, Kharagpur during 1963 - 1998. He guided $14 \mathrm{PhDs}$, and published more than 86 research papers in various international journals. He received the best Engineering College Faculty Award in Computer Science and Engineering for the year 2008 from the Indian Society for Technical

Education (AP Chapter), Best Teacher Award by Lions Clubs International, Hyderabad Elite, in 2012, and CognizantSreenidhi Best faculty award for the year 2012. His research interests are Network Security \& Cryptography, Image Processing, Data Mining and Genetic Algorithms.

K. Shirisha is currently working as Associate Professor in the Department of Computer Science and Engineering (CSE), SreeNidhi Institute of Science \& Technology (SNIST), Hyderabad, India, since February 2007. She is pursuing her Ph.D. Her research interests are Data Mining and Information Security. 